\title{
New opportunities for tensor-free calculations of residual dipolar couplings for the study of dynamic nuclear polarization of nucleic acids with endogenously bound manganese in gum cancer cells
}

\author{
Alireza Heidari ${ }^{1,2 *}$ and Ricardo Gobato ${ }^{3}$ \\ ${ }^{1}$ Faculty of Chemistry, California South University, 14731 Comet St. Irvine, CA 92604, USA \\ ${ }^{2}$ American International Standards Institute, Irvine, CA 3800, USA \\ ${ }^{3}$ Green Land Landscaping and Gardening, Seedling Growth Laboratory, 86130-000, Parana, Brazil
}

\begin{abstract}
In the current study, new opportunities for tensor-free calculations of residual dipolar couplings for the study of dynamic nuclear polarization of nucleic acids with endogenously bound manganese in gum cancer cells are investigated. Tensor-free calculations of residual dipolar couplings for the study of dynamic nuclear polarization of nucleic acids with endogenously bound manganese in gum cancer cells originates initially from analytical chemistry to evaluate chemical compounds based on varied excitation of vibrational modes in the internal chemical bonds. Since tensor-free calculations of residual dipolar couplings for the study of dynamic nuclear polarization of nucleic acids with endogenously bound manganese in gum cancer cells is able to detect tensor-free calculations of residual dipolar couplings for the study of dynamic nuclear polarization of nucleic acids with endogenously bound manganese in gum cancer cells signals from the bonds within molecules, it can also provide the biochemical conditions within biological samples. Within a molecularly complicated biological system, like a cell, tissue or even an organ, tensor-free calculations of residual dipolar couplings for the study of dynamic nuclear polarization of nucleic acids with endogenously bound manganese in gum cancer cells carries intrinsic details and information of the materials present in the system, thus the biological status can also be acquired based on which the detailed features of the tissue are easily and accurately obtained.
\end{abstract}

\section{Introduction}

Tensor-free calculations of residual dipolar couplings for the study of dynamic nuclear polarization of nucleic acids with endogenously bound manganese in gum cancer cells is a spectroscopic technique capable of providing highly detailed chemical information about a tissue sample. In contrast to other optical spectroscopic techniques, there are a large number of tensor-free calculations of residual dipolar couplings for the study of dynamic nuclear polarization of nucleic acids with endogenously bound manganese in gum cancer cells in gum cancer tissue and their spectral signatures are sharp and well delineated. The ability to probe several different chemicals is of particular importance in studying gum cancer, due to the heterogeneity of disease. Further, tensor-free calculations of residual dipolar couplings for the study of dynamic nuclear polarization of nucleic acids with endogenously bound manganese in gum cancer cells is particularly amenable to in vivo measurements as the powers and excitation wavelengths used are non-destructive to the tissue and have a relatively large penetration depth. For these reasons, we have investigated the use of tensor-free calculations of residual dipolar couplings for the study of dynamic nuclear polarization of nucleic acids with endogenously bound manganese in gum cancer cells as a clinical tool for the examination of a variety of pathologies [1-10].

\section{Results and discussion}

Bladder gum cancer is one of the severest human malignancies which are hardly detected at an early stage. Tensor-free calculations of residual dipolar couplings for the study of dynamic nuclear polarization of nucleic acids with endogenously bound manganese in gum cancer cells is reported to maintain a high diagnostic accuracy, sensitivity and specificity in some tumors. Both tensor-free calculations of residual dipolar couplings for the study of dynamic nuclear polarization of nucleic acids with endogenously bound manganese in gum cancer cells and surface enhanced tensor-free calculations of residual dipolar couplings for the study of dynamic nuclear polarization of nucleic acids with endogenously bound manganese in gum cancer cells are proving to be invaluable tools in the field of biomedical research and clinical diagnostics. The robust, compact, fit-for-purpose tensor-free calculations of residual dipolar couplings for the study of dynamic nuclear polarization of nucleic acids with endogenously bound manganese in gum cancer cells designs are appropriate for use in surgical procedures to help surgeons assess tumors and allow rapid

*Correspondence to: Alireza Heidari, Faculty of Chemistry, California South University, 14731 Comet St. Irvine, CA 92604; American International Standards Institute, Irvine, CA 3800, USA, E-mail: Scholar.Researcher.Scientist@gmail. com; Alireza.Heidari@calsu.us; Central@aisi-usa.org

Key words: diagnosis, gum cancer cells, DNA/RNA, database mining, regression, high resolution, assignment

Received: October 23, 2020; Accepted: November 23, 2020, Published: November 27, 2020 
Heidari A (2020) New opportunities for tensor-free calculations of residual dipolar couplings for the study of dynamic nuclear polarization of nucleic acids with endogenously bound manganese in gum cancer cells

decisions to be made. Tensor-free calculations of residual dipolar couplings for the study of dynamic nuclear polarization of nucleic acids with endogenously bound manganese in gum cancer cells are also being developed for molecular diagnostic testing to detect and measure human gum cancer biomarkers. Based on the SERS technique, this approach potentially could change the way bioassays are performed to improve both the sensitivity and reliability of testing. The two applications highlighted in this review, together with other examples of the use of tensor-free calculations of residual dipolar couplings for the study of dynamic nuclear polarization of nucleic acids with endogenously bound manganese in gum cancer cells in biomedical research areas such as the identification of bacterial infections, are clearly going to make the technique an important part of the medical toolbox, as we continually strive to improve diagnostic techniques and bring a better health care system to patients (Figure 1).

\section{Conclusion}

Because of the advantages of tensor-free calculations of residual dipolar couplings for the study of dynamic nuclear polarization of nucleic acids with endogenously bound manganese in gum cancer cells, an increased appliance of tensor-free calculations of residual dipolar couplings for the study of dynamic nuclear polarization of nucleic acids with endogenously bound manganese in gum cancer cells in the early and precise diagnosis of $\mathrm{BCa}$ has been reported. Meanwhile, a large number of clinical trials studying the possibility of using tensor-free calculations of residual dipolar couplings for the study of dynamic nuclear polarization of nucleic acids with endogenously bound manganese in gum cancer cells on epithelial cells from voided urines to detect early-stage malignancies have been carried out. However, results

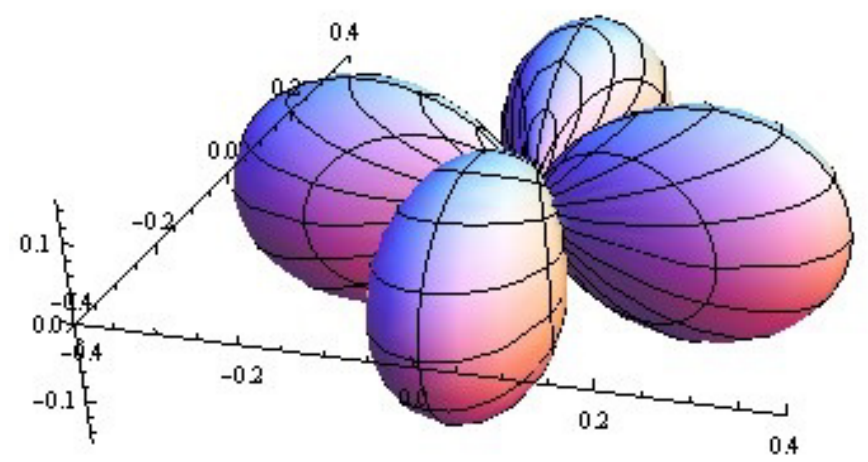

Figure 1. Tensor-free calculations of residual dipolar couplings for the study of dynamic nuclear polarization of nucleic acids with endogenously bound manganese in gum cancer cells from different studies vary from each other. This can result from different numbers of people recruited, different nationalities of the people recruited or regional variations, etc. Thus, a comprehensive research to integrate the already published studies to acquire the most accurate and reliable data is proposed. This systematic review and meta-analysis aims to integrate these studies and come up with results depleted of potential errors and bias among them to provide the most reliable data concerning the efficiency of applying tensor-free calculations of residual dipolar couplings for the study of dynamic nuclear polarization of nucleic acids with endogenously bound manganese in gum cancer cells in the diagnosis of BCa.

\section{Acknowledgments}

This study was supported by the Cancer Research Institute (CRI) Project of Scientific Instrument and Equipment Development, the National Natural Science Foundation of the United Sates, the International Joint BioSpectroscopy Core Research Laboratory Program supported by the California South University (CSU), and the Key project supported by the American International Standards Institute (AISI), Irvine, California, USA.

\section{References}

1. Heidari A, Brown C (2015) Study of composition and morphology of cadmium oxide (CdO) nanoparticles for eliminating cancer cells. J Nanomed Res 2(5): 20.

2. Heidari A, Brown C (2015) Study of surface morphological, phytochemical and structural characteristics of rhodium (III) oxide $\left(\mathrm{Rh}_{2} \mathrm{O}_{3}\right)$ nanoparticles. International Journal of Pharmacology, Phytochemistry and Ethnomedicine 1(1): 15-19.

3. Heidari A (2016) An experimental biospectroscopic study on seminal plasma in determination of semen quality for evaluation of male infertility. Int $J$ Adv Technol 7: e007.

4. Heidari A (2016) Extraction and preconcentration of N-Tolyl-Sulfonyl-PhosphoramidSaeure-Dichlorid as an anti-cancer drug from plants: a pharmacognosy study. $J$ Pharmacogn Nat Prod 2: e103.

5. Heidari A (2016) A thermodynamic study on hydration and dehydration of DNA and RNA-Amphiphile Complexes. J Bioeng Biomed Sci S: 006.

6. Heidari A (2016) Computational studies on molecular structures and carbonyl and ketene groups' effects of singlet and triplet energies of Azidoketene $\mathrm{O}=\mathrm{C}=\mathrm{CH}-\mathrm{NNN}$ and Isocyanatoketene $\mathrm{O}=\mathrm{C}=\mathrm{CH}-\mathrm{N}=\mathrm{C}=\mathrm{O} . J$ Appl Computat Math 5: e142.

7. Heidari A (2016) Study of irradiations to enhance the induces the dissociation of hydrogen bonds between peptide chains and transition from helix structure to random coil structure using ATR-FTIR, Raman and ${ }^{~}$ HNMR Spectroscopies. J Biomol Res Ther 5: e146.

8. Heidari A (2016) Future prospects of point fluorescence spectroscopy, fluorescence imaging and fluorescence endoscopy in photodynamic therapy (PDT) for cancer cells. J Bioanal Biomed 8: e135.

9. Heidari A (2016) A bio-spectroscopic study of DNA density and color role as determining factor for absorbed irradiation in cancer cells. Adv Cancer Prev 1: e102.

10. Heidari A (2016) Manufacturing process of solar cells using cadmium oxide (CdO) and rhodium (III) Oxide $\left(\mathrm{Rh}_{2} \mathrm{O}_{3}\right)$ nanoparticles. J Biotechnol Biomater 6: e125.

Copyright: (C2020 Heidari A. This is an open-access article distributed under the terms of the Creative Commons Attribution License, which permits unrestricted use, distribution, and reproduction in any medium, provided the original author and source are credited. 